\title{
Fast Resolution of Recurrent Pronounced Macular Edema following Intravitreal Injection of Dexamethasone $0.7 \mathrm{mg}$
}

\author{
Linda M. Meyer Carl-Ludwig Schönfeld \\ Herzog Carl-Theodor Eye Clinic, Munich, Germany
}

\section{Key Words}

Central retinal vein occlusion · Dexamethasone $0.7 \mathrm{mg} \cdot$ Ozurdex $^{\circledR} \cdot$ Recurrent macular edema

\begin{abstract}
Purpose: To report the fast resolution of recurrent pronounced macular edema due to central retinal vein occlusion (CRVO) within $72 \mathrm{~h}$ following intravitreal injection of dexamethasone $0.7 \mathrm{mg}\left(\mathrm{Ozurdex}^{\circledR}\right)$.

Methods: An interventional case report with optical coherence tomography scans and fluorescein angiographic pictures.
\end{abstract}

Results: A 69-year-old Caucasian man underwent intravitreal injection of dexamethasone $0.7 \mathrm{mg}$ due to incomplete CRVO. He had previously undergone 6 intravitreal injections of bevacizumab $1.25 \mathrm{mg}$ (Avastin ${ }^{\circledR}$ ) and a C-grid laser photocoagulation over an interval of 16 months. After repeated recurrences of macular edema, the injection of dexamethasone reduced the macular edema from $570 \mu \mathrm{m}$ preoperatively to $246 \mu \mathrm{m}$ postoperatively within $72 \mathrm{~h}$ following the injection. Best-corrected visual acuity improved from 0.1 to 0.6 within the same interval.

Conclusion: Dexamethasone can lead to a very fast reduction of macular edema in patients with vision loss due to CRVO and may facilitate an immediate visual rehabilitation. Retinal anatomy and visual acuity may be restored even in long-standing, recurrent cases.

\section{Introduction}

Macular edema is the most common cause of visual loss in patients with central retinal vein occlusion (CRVO) and branch retinal vein occlusion (BRVO) [1-3]. Even though the pathogenesis of macular edema in retinal occlusive vascular disease is not yet fully 
understood, the presence of inflammatory cytokines as well as vascular permeability factors, such as interleukin-6, prostaglandins, and VEGF, and the consecutive breakdown of the blood-retina barrier due to endothelial cell dysregulation are postulated to cause macular edema $[4,5]$. Dexamethasone has potent anti-inflammatory properties and has been shown to be a favorable treatment option in patients with CRVO and BRVO [6]. So far, it is unknown how fast the macular edema can be successfully treated with dexamethasone $0.7 \mathrm{mg}$. We present a case of complete resolution of extensive recurrent macular edema and significant improvement of best-corrected visual acuity (BCVA) within $72 \mathrm{~h}$ of intravitreal implantation of dexamethasone $\left(\right.$ Ozurdex $\left.^{\circledR}\right)$.

\section{Case Report}

In 2009, a 69-year-old man presented for the first time with impaired vision (BCVA 0.5) due to a CRVO with a macular edema in his right eye. Following intravitreal injection of bevacizumab $1.25 \mathrm{mg}$ $\left(\right.$ Avastin ${ }^{\circledR}$ ), his vision improved to 1.0. Five weeks later, his BCVA had dropped to 0.6. Again, he underwent intravitreal injection of bevacizumab with prompt functional improvement to BCVA 0.8. Over a period of 8 months, he experienced chronic recurring macular edema and received 4 further injections of bevacizumab in total. The last treatment was supplemented with a C-grid laser photocoagulation ( $\mathrm{n}=93$ effects) 4 weeks following the last injection. At that time, the macular edema had resolved completely and vision had stabilized at 0.8 . However, the macular edema recurred again 2 weeks after the laser treatment as shown in figure 1 with fluorescein angiography and optical coherence tomography (OCT). At this time point, the central retinal thickness was $326 \mu \mathrm{m}$, and BCVA had dropped to 0.5 . Now, the patient was treated for the first time with dexamethasone intravitreal implant (Ozurdex). Four weeks after dexamethasone implantation, his BCVA had improved to 0.8, and the OCT scan revealed a resolved macular edema. Three months later, the macular edema recurred again. BCVA had dropped to 0.1 , and the OCT scan revealed a pronounced macular edema with a central retinal thickness of $570 \mu \mathrm{m}$ (fig. 2). The patient again received dexamethasone intravitreal implant in his right eye. Seventy-two hours following this second injection of Ozurdex, his BCVA had improved to 0.6, and the pronounced macular edema had resolved completely ( $\underline{\text { fig. }}$ ) $)$. Central retinal thickness had normalized to $246 \mu \mathrm{m}$. The patient's condition has so far been stable for at least 12 weeks.

\section{Discussion}

Recently, it has been shown that dexamethasone in a biodegradable drug delivery system (Ozurdex) can improve visual acuity and reduce macular edema in patients with vision loss due to retinal vein occlusion [6]. However, so far no information is available on how fast the macular edema can be antagonized by the dexamethasone implant. The data presented in this case report indicate that the dexamethasone implant has an immediate effect on macular edema due to retinal vein occlusion that sets in within the first $72 \mathrm{~h}$ following the intravitreal implantation of the drug. A possible explanation of this fast effect may be the relatively high intraocular dose of dexamethasone that is released from the implant. The effect of degenerative processes at the level of the retinal pigment epithelium (fig. 3), which might set in even though macular edema is resolved by the dexamethasone implant, need to be examined in the near future.

In conclusion, the dexamethasone implant (Ozurdex) may lead to fast functional improvement and visual rehabilitation in patients with macular edema due to retinal vein occlusion even in recurrent or long-standing cases. 


\section{Disclosure Statement}

The authors have no conflicts of interest.

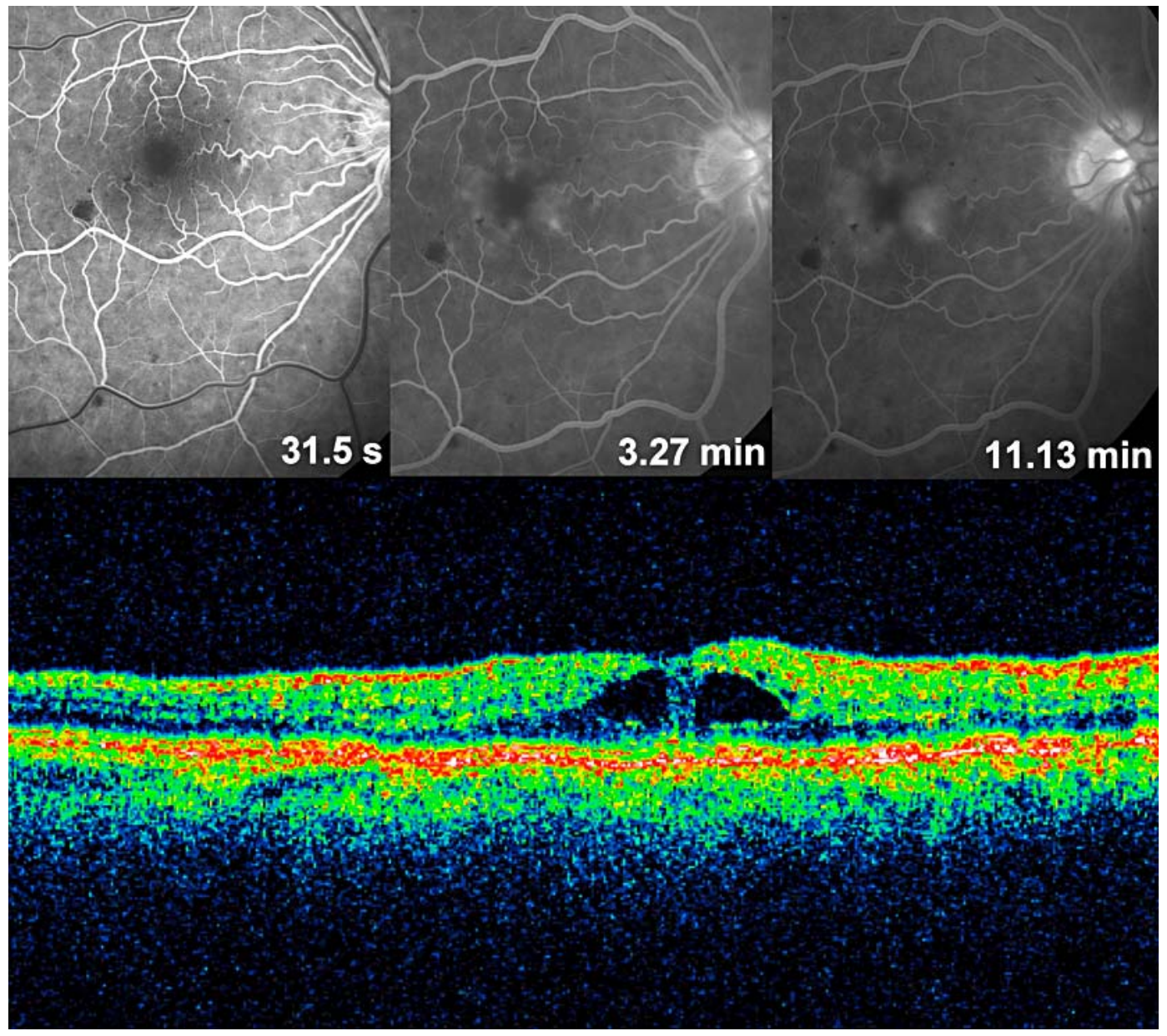

Fig. 1. Fluorescein angiography and OCT scan of recurrent macular edema following repeated intravitreal injections of bevacizumab and focal C-grid laser photocoagulation of the macula. 


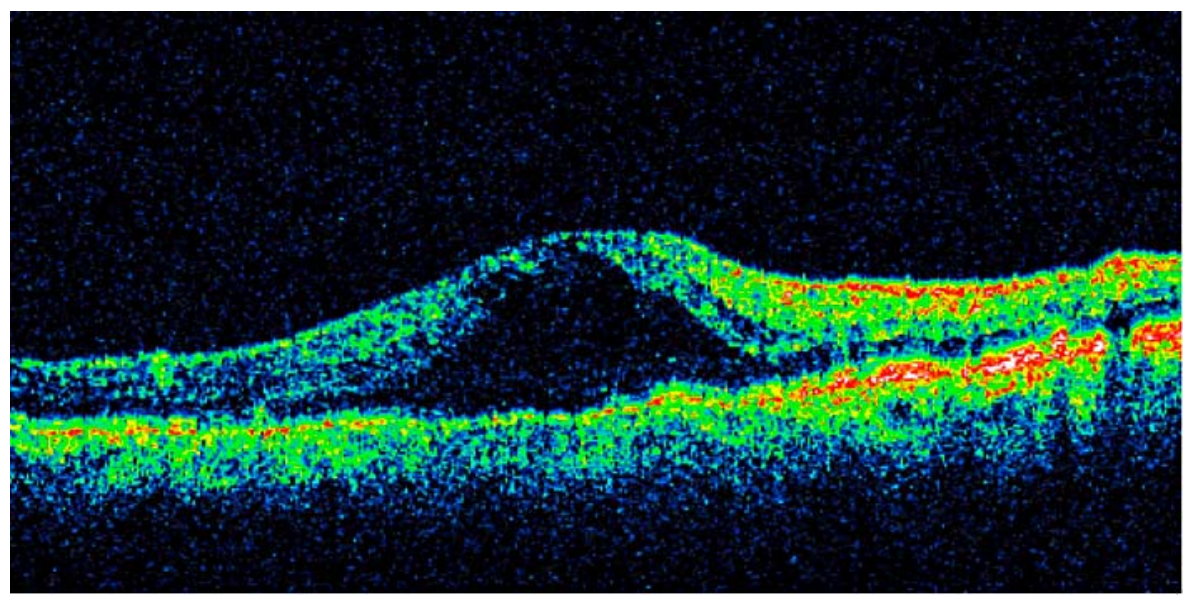

Fig. 2. OCT scan of recurrent macular edema 3 months following repeated intravitreal injections of bevacizumab, C-grid laser photocoagulation and 1 intravitreal injection of dexamethasone $0.7 \mathrm{mg}$ (Ozurdex). BCVA had dropped to 0.1. Central retinal thickness was $570 \mu \mathrm{m}$.

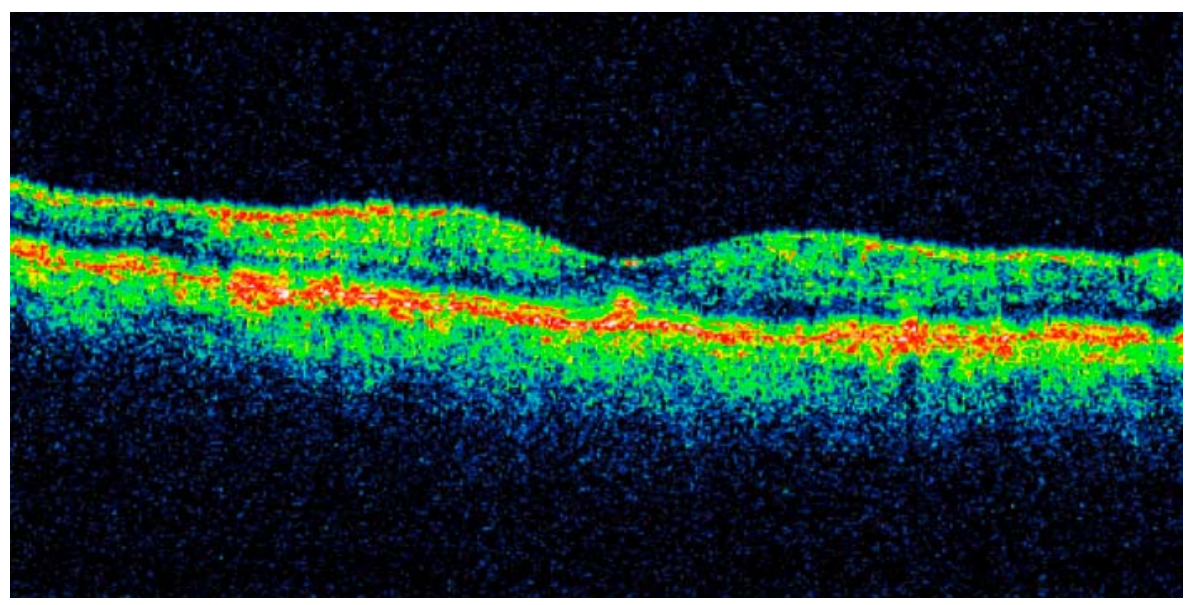

Fig. 3. OCT scan of resolved macular edema $72 \mathrm{~h}$ following the second intravitreal injection of dexamethasone $0.7 \mathrm{mg}$ (Ozurdex). BCVA was 0.6. Central retinal thickness was $246 \mu \mathrm{m}$. In the outer layers, degenerative processes are visible at the level of the retinal pigment epithelium. 


\section{References}

1 Zegarra H, Gutman FA, Conforto J: The natural course of central vein occlusion. Ophthalmology 1979;86:1931-1942.

2 Mitchell P, Smith W, Chang A: Prevalence and associations of retinal vein occlusion in Australia: the Blue Mountains Eye Study. Arch Ophthalmol 1996;114:1243-1247.

-3 Rehak J, Rehak M: Branch retinal vein occlusion: pathogenesis, visual prognosis, and treatment modalities. Curr Eye Res 2008;33:111-131.

-4 Campochiaro PA, Hafiz G, Shah SM, et al: Ranibizumab for macular edema due to retinal vein occlusions; implication of VEGF as a critical stimulator. Mol Ther 2008;16:791-799.

5 Chen KH, Wu CC, Roy S, Lee SM, Liu JH: Increased interleukin-6 in aqueous humor of neovascular glaucoma. Invest Ophthalmol Vis Sci 1999;40:2627-2632.

6 Haller JA, Bandello F, Belfort R Jr, et al: OZURDEX GENEVA Study Group. Randomized, sham-controlled trial of dexamethasone intravitreal implant in patients with macular edema due to retinal vein occlusion. Ophthalmology 2010;117:1134-1146. 